\title{
ARTÍGULO ORIGINAL: Evaluación de resultados en el uso de la plasmaféresis. Servicio de Cuida- dos Intensivos, Hospital Dr. Rafael Ángel Calde- rón Guardia. Enero 2010 a Diciembre 2012
}

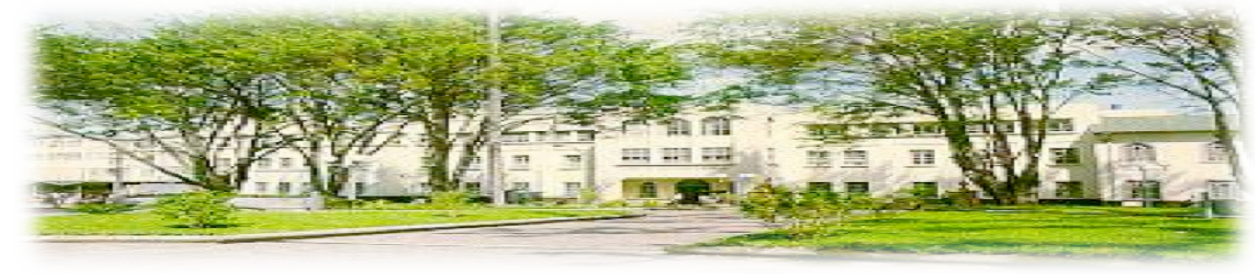

Hospital San quan de Dias. San José, Casta Rica. Fundado en 1845
Recibido: Aceptado:
$18 / 01 / 2014$

$21 / 04 / 2014$
Juan Richmond Navarro ${ }^{1}$ Karla Quesada Ureña ${ }^{2}$

\footnotetext{
${ }^{\mathbf{1}}$ Especialista en Medicina Interna y Hematología. Servicio de Hemato-Oncología. Hospital Dr. Rafael Ángel Calderón Guardia. C.C.S.S. San José Costa Rica.

${ }^{2}$ Especialista en Medicina Interna. Hospital Dr. Rafael Ángel Calderón Guardia. C.C.S.S. San José. Costa Rica.
}

\section{RESUMEN}

Introducción: El objetivo principal de este estudio fue describir los resultados de la aplicación de esta terapia en los pacientes ingresados en la Unidad de Cuidados Intensivos del Hospital Dr. Rafael Ángel Calderón Guardia. Materiales y Métodos: estudio observacional, descriptivo y retrospectivo desarrollado mediante la revisión de expedientes de pacientes mayores de 18 años ingresados en la UCI, a los que se les realizó terapia de aféresis. Se procedió al llenado de la hoja de recolección de datos desarrollada para este fin. Se incluyó 50 pacientes que cumplieron los criterios de inclusión e ingresaron a la UCI en el periodo de estudio. Resultados: del total de los pacientes ingresados para terapia de aféresis un $40 \%$ correspondió a patología neurológica; de estos, un $20 \%$ ingresó por Síndrome de Guillian-Barré, 30\% por patologías hematológicas (principalmente AHAI), un 18\% por pancreatitis no biliar a asociada a hipertrigliceridemia severa y el resto correspondió a patologías reumatológicas. El $80 \%$ de los pacientes ingresados recibió de una a siete sesiones de aféresis independiente de la patología de ingreso a la UCI. Al momento del ingreso a UCI, un $96 \%$ de los pacientes presentó compromiso neurológico, de los cuales lo más frecuente fue la debilidad motora progresiva reportada en un $82 \%$ de los casos; un $86 \%$ de los ingresos con compromiso respiratorio, los cuales ameritaron soporte ventilatorio; y un $92 \%$ de los casos con compromiso renal requirió algún tipo de soporte renal. Un $84 \%$ de los casos fue egresado de la UCI aún con compromiso neurológico, siendo la debilidad motora progresiva el parámetro con mayor beneficio, con un 54\%. Un 98\% de los egresados continuó con soporte ventilatorio y un $94 \%$ con algún tipo 
de soporte renal. Cuarenta y cinco pacientes (90\%) ingresaron con compromiso hematológico, y a su egreso de la unidad 33 pacientes $(66 \%)$ presentaron mejoría con respecto al ingreso. En cuanto a la función renal de estos pacientes, $8 \mathrm{de}$ ellos, correspondiente al 16\%, ingresaron con creatininas mayores a $2 \mathrm{mg} / \mathrm{dl}$ y a al egreso, 7 pacientes (14\%) mantuvieron iguales los niveles que al ingreso. El promedio del tiempo de hospitalización fue de 90.7 días, con una mediana de 36.5 días de estancia hospitalaria. Conclusiones: el $60 \%$ de los casos que ingresó a la UCI para terapia de aféresis correspondió a patología neurológica, siendo el Guillain-Barré el de mayor prevalencia. Un 30\% ingresó por patología hematológica que requirió aféresis y un $18 \%$ por pancreatitis no biliar secundaria a hipertrigliceridemia severa. Se encontró mejoría en parámetros clínicos y de laboratorio en la mayoría de los criterios de ingreso de los pacientes al comparar los mismos al egreso de la unidad.

\section{PALABRAS CLAVE}

Plasmaferesis. Aféresis. Síndrome de GuillainBarré. Miastenia gravis. Esclerosis Múltiple. Púrpura Trombocitopénica Trombótica. Anemia Hemolítica Autoinmune. Hipertrigliceridemia severa. Pancreatitis. Hiperviscocidad.

\section{ABSTRACT}

Introduction: The objective of the study is to describe the results of the application of this therapy in patients admitted to the Intensive Care Unit of the Dr. Rafael Ángel Calderón Guardia Hospital. Materials and Methods: This was an observational, descriptive and retrospective study conducted by reviewing records of patients over 18 years admitted to the ICU, who underwent apheresis therapy. Proceeded to fill the data collection sheet developed for this purpose. 50 patients who met the inclusion criteria were included and admitted to the ICU in the study period. Results: of all patients admitted to apheresis therapy, $40 \%$ were cases of neurological diseases, of these, 20\% had Guillain-Barré syndrome, 30\% have hematologic diseases (mainly autoimmune hemolytic anemia), $18 \%$ of the admissions to the ICU were by non-biliary pancreatitis associated with severe hypertriglyceridemia and the rest were reported by rheumato- logic diseases. $80 \%$ of admitted patients received one to seven sessions of apheresis independent of the pathology that require the ICU admission. At admission to the ICU, $96 \%$ of the patients had neurological involvement, being progressive motor weakness the parameter more reported in $82 \%$ of cases, $86 \%$ of the admissions had respiratory compromise, which require ventilator support, and $92 \%$ of patients with renal involvement required some form of renal support. $84 \%$ of patients were discharged from the ICU with neurological involvement, being progressive motor weakness the parameter with greater benefit, with $54 \%$. $98 \%$ of the patients discharged continued with ventilatory support and $94 \%$ continued with some form of renal support. Forty-five patients $(90 \%)$ were admitted with hematological compromise, at discharge from the unit, thirty-three patients $(66 \%)$ showed improvement with respect to the admission. As for the renal function of these patients, 8 of them admitted, corresponding to $16 \%$, had creatinine levels $>2$ $\mathrm{mg} / \mathrm{dl}$, and at discharge, seven patients (14\%) maintained the same income levels. The average hospital stay was 90.7 days, with a median of 36.5 days. Conclusions: $60 \%$ of patients admitted to the ICU for apheresis therapy correspond to neurological disease, being the most prevalent etiology Guillain-Barré syndrome. 30\% admitted for hematological pathology require apheresis and $18 \%$ for non-biliary pancreatitis secondary to severe hypertriglyceridemia. Improvement was found in clinical and laboratory parameters in most of the criteria for admission of patients when compare themselves to discharge from the unit.

\section{KEY WORDS}

Plasmapheresis. Guillain-Barré Syndrome. Miastenia Gravis. Multiple Sclerosis. Thrombotic Thrombocytopenic Purpura. Autoimmune hemolytic anemia. Severe Hypertriglyceridemia. Pancreatitis. Hyperviscosity.

\section{INTRODUCCIÓN}

La aféresis terapéutica es el procedimiento que separa y remueve el plasma de la sangre total, eliminando así componentes considerados responsables patógenos de una enfermedad o de sus manifestaciones clínicas ${ }^{(8)}$. 
Las bases actuales de la aféresis terapéutica, fueron descritas por primera vez en 1914 por Abel, Rowentree y Turner, en la Universidad de Johns Hopkins; al describir la técnica de la plasmaféresis. La aplicación de esta técnica inicial fue bastante limitada debido a que no se contaba aún con la maquinaria automática específica para separar los diferentes componentes de la sangre $^{(8)}$.

Durante los años 60, se consolidó la técnica de aféresis, con el desarrollo de las membranas separadoras del plasma, base científica de las nuevas membranas de hemofiltración ${ }^{(7)}$.

El mecanismo por el cual la plasmaferesis resulta efectiva, es mediante la depleción rápida de factores específicos asociados a la enfermedad; los cuales incluyen auto-anticuerpos patogénicos tipo inmunoglobulinas $\mathrm{G}$ y $\mathrm{M}$, complejos inmunes circulantes, crioglobulinas, cadenas ligeras de inmunoglobulinas y lipoproteínas con alto contenido de colesterol $^{(7)}$. El objetivo principal es remover estas sustancias y permitir la reversión del proceso patológico así como la disminución de sus manifestaciones clínicas ${ }^{(7)}$.

Se ha descrito otros mecanismos, por ejemplo la sustitución de factores deficitarios del plasma, ya que se al remover el plasma del paciente, es reemplazado por plasma normal, administrando elementos deficitarios como una metaloproteasa de adhesión del factor von Willebrand (ADAMTS-13) $^{(3,4)}$.

La aféresis tiene efecto en la modulación de la respuesta inmune, desbloqueando al sistema reticuloendotelial y mejorando la depuración endógena de anticuerpos o complejos inmunes, mediante remoción de mediadores inflamatorios (citoquinas-complemento) y estimulación de clones de linfocitos para mejorar la respuesta citotóxica $^{(7)}$.

A nivel Internacional, la Asociación Americana para Aféresis (ASFA) y la Asociación Americana de Bancos de Sangre (AABB) actualizaron las guías clínicas para el uso de aféresis terapéutica en la práctica clínica, con un abordaje basado en la evidencia de la Sociedad América de Aféresis, dividiendo las recomendaciones en 4 categorías ${ }^{(6)}$.

La Primera Categoría, incluye patologías en las que la aféresis es aceptada como primera línea de tratamiento, ya sea como primer escalón o en conjunto con otras opciones de tratamiento; por ejemplo plasma separación en el Síndrome Guillain-Barré (como terapia de primera línea utilizada sola) y en Miastenia Gravis (como primera línea utilizada con terapia inmunosupresora e inhibición de la colinesterasa).

En la Segunda Categoría, se incluye patologías en las que la plasmaféresis es aceptada como segunda línea de tratamiento, ya sea como única opción o asociada a otras modalidades de tratamiento; como por ejemplo la plasma separación en casos de encefalomielits aguda diseminada posterior a dosis altas de esteroides intraveno$\operatorname{sos}^{(6)}$.

En la Tercera Categoría, se incluye aquellas en donde el rol óptimo de la aféresis no se ha establecido aún; por lo que la aplicación de esta terapéutica debe ser individualizada, como en casos de pacientes con sepsis y falla multiorgáni$\mathrm{ca}^{(6)}$.

La Cuarta Categoría, se refiere a patologías en las cuales la evidencia publicada demuestra o sugiere que el uso de aféresis es inefectiva o puede inducir daño, mencionando como ejemplo, casos de artritis reumatoide $\operatorname{activa}^{(6)}$.

A nivel nacional, se carece de información así como lineamientos y/o protocolos, que permitan una intervención apropiada en beneficio del paciente, al proponer una intervención rápida $\mathrm{y}$ efectiva con disminución de las complicaciones propias de la enfermedad.

El objetivo de este estudio es describir los resultados de la plasmaféresis utilizada en pacientes de la Unidad de Cuidados Intensivos, del Hospital Dr. Rafael Ángel Calderón Guardia, de Enero 2010 a Diciembre 2012, mediante la documentación de los resultados positivos y complicaciones del uso de este procedimiento de acuerdo a las diferentes patologías.

\section{MATERIALES Y MÉTODOS}

El presente es un estudio observacional, descriptivo y retrospectivo desarrollado mediante la revisión de expedientes de pacientes que ingresaron a la Unidad de Cuidados Intensivos del Hospital Rafael Ángel Calderón Guardia, a los cuales se les practicó terapia de aféresis, en un periodo 
comprendido de enero del 2010 a diciembre del 2012 .

Se procedió al llenado de la hoja de recolección de datos con el uso del expediente clínico. Los criterios de inclusión correspondieron a todos los pacientes mayores de 18 años ingresados en la Unidad de Cuidados Intensivos del Hospital R.A Calderón Guardia, en los que se utilizó terapia de plasmaféresis de enero 2010 a Diciembre 2012; no hubo restricción por género, etnia y terapia farmacológica concomitante.

Se excluyó los individuos en los cuales se realizó terapia de aféresis fuera de la Unidad de Cuidados Intensivos del Hospital Calderón Guardia y posteriormente fueron ingresados para monitoreo; así como también los pacientes menores de 18 años.

Análisis estadístico: la información recopilada se digitó en una base de datos elaborada para este estudio, siendo el paciente la unidad de análisis. Consistió de 50 pacientes. Una vez llena, se realizó pruebas de inconsistencia y errores. Los datos se procesaron en el paquete estadístico SPSS versión 2.1 y Excel para la elaboración de los cuadros.

Se analizó variables cualitativas: compromiso neurológico, ventilatorio y renal; determinadas por una escala subjetiva, independiente de la causa que originara la utilización de soporte tanto ventilatorio como renal y variables cuantitativas, las cuales correspondieron a parámetros de laboratorio.

Se realizó frecuencias simples de todas las variables, para las variables cuantitativas además se obtuvo medidas de tendencia central y dispersión.

El comportamiento de las variables: compromiso neurológico, compromiso respiratorio, compromiso renal, hemoglobina, plaquetas, reticulocitos, nitrógeno ureico, creatinina, LDH y triglicéridos fueron comparados en los pacientes según los datos de ingreso y egreso.

Los resultados de las comparaciones se sometieron a la prueba de significancia estadística de Chi-cuadrado para la comparación de los cambios porcentuales después del tratamiento (ingreso y egreso), tanto para las variables nominales como las numéricas, dado que estas últimas se analizaron en clases.

Se consideró significativo un nivel de confianza $<0.05$.

Este estudio fue avalado por el Comité Local de Bioética e Investigación del Hospital Rafael Ángel Calderón Guardia.

\section{RESULTADOS}

Durante la revisión inicial se obtuvo un total de 78 pacientes con ingreso a la Unidad de Cuidados Intensivos del Hospital, para el periodo de 2010 a 2012, a los que se les realizó terapia de aféresis.

De la totalidad de expedientes, 27 casos fueron excluidos (15 expedientes no fueron localizados, 8 pacientes habían recibido sesiones de aféresis en otros servicios y fueron ingresados a UCI para monitoreo de otras complicaciones, 3 pacientes eran menores de 18 años). Se incluyó 50 pacientes que cumplieron los criterios de inclusión e ingresaron a la Unidad de Cuidados Intensivos en el periodo de estudio.

Un $56 \%$ de los pacientes fueron mujeres. La edad promedio de los entrevistados fue de 38.9 años, con una desviación estándar de 16 y una mediana de 36.5. Agrupándolos por edades, la mayor parte del grupo correspondió al de 30 a 39 años con un $22 \%$, mientras que solo el $12 \%$ fueron mayores de 60 años.

Con respecto al área de atracción, se identificó 3 localidades con la mayoría de los casos ingresados, con un $16 \%$ cada una, correspondientes a Guadalupe, Curridabat y Coronado; solamente un $2 \%$ de los casos no pertenecían al área de atracción, teniendo como domicilio Golfito.

De los pacientes ingresados a la Unidad de Cuidados Intensivos para recibir terapia de aféresis, 20 pacientes $(40 \%)$ ingresaron por patología neurológica; de estos, 10 individuos $(20 \%)$ portadores de Síndrome de Guillain-Barré; 5 pacientes $(10 \%)$ presentaron miastenia gravis y otros 5 (10\%), esclerosis múltiple. (Gráfico 1). Un 30\% de los ingresos para aféresis correspondió a patologías hematológicas, de éstas la más frecuente fue la anemia hemolítica autoinmune, con un 16\% de los casos. (Gráfico 1). 
Gráfico 1. Porcentaje de pacientes ingresados en la Unidad de Cuidados Intensivos del Hospital Dr. Rafael Ángel Calderón Guardia, a los que se les realizó terapia de aféresis, según enfermedad de base, del 01 de Enero del 2010 al 31 de Diciembre del 2012.

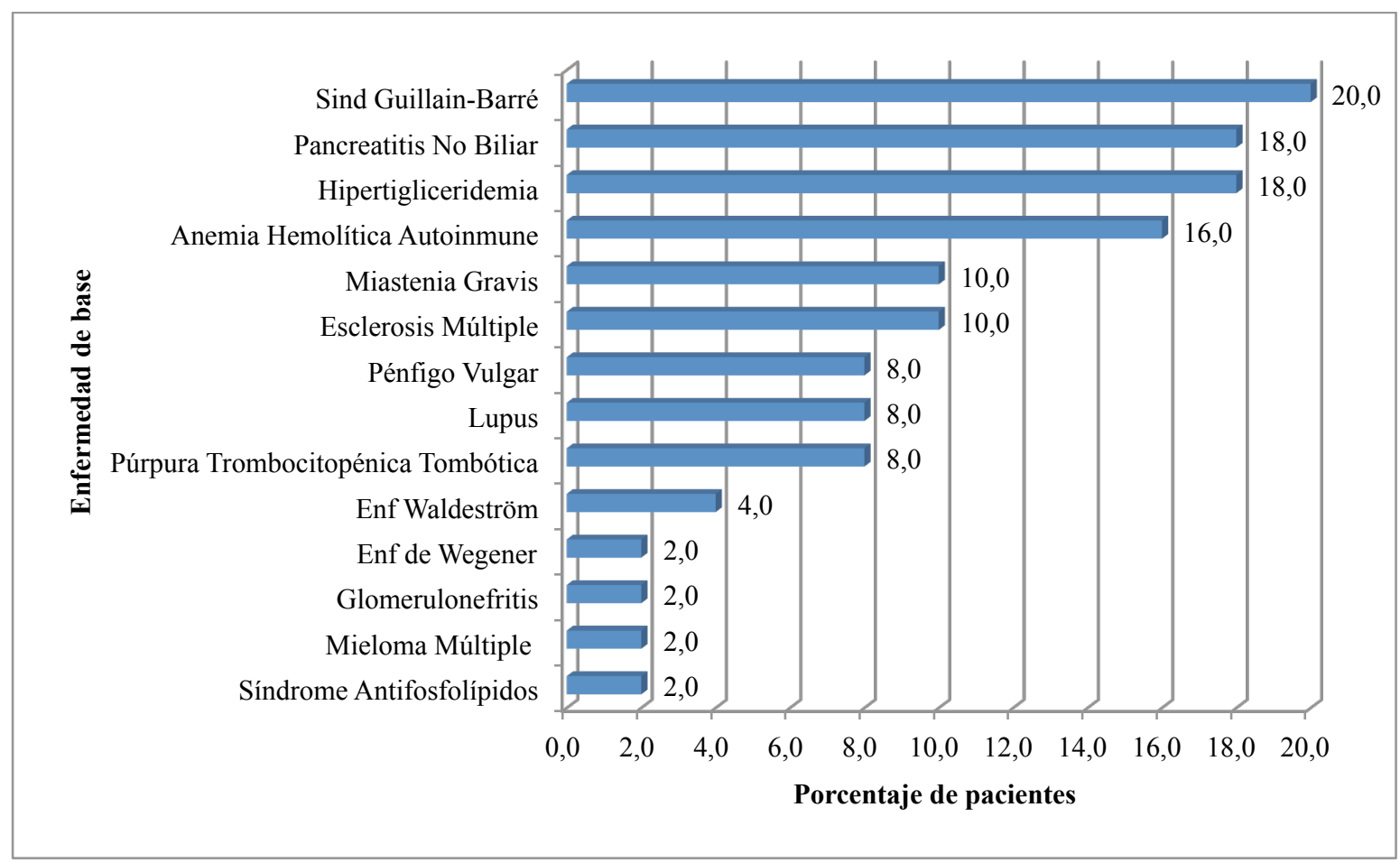

Fuente: Expedientes Clínicos, HCG.

En tercer lugar de frecuencia de ingresos, se documentó con un $18 \%$, los casos por pancreatitis no biliar asociada a hipertrigliceridemia severa. (Gráfico 1).

En lo que respecta a patologías con compromiso reumatológico, ingresaron 6 pacientes; documentando un $12 \%$ de los casos, incluyendo pacientes portadores de lupus eritematoso sistémico, síndrome antifosfolípidos catastrófico y enfermedad de Wegener. (Gráfico 1).

Un $8 \%$ de los casos correspondió a patología dermatológica, representada por Pénfigo Vulgar. Únicamente un $2 \%$ de los casos respectivamente correspondió a enfermedades con compromiso renal. (Gráfico 1).

Cuarenta pacientes, correspondiente al $80 \%$ de la población estudiada, recibieron de una a siete sesiones independiente de la patología de ingreso a la UCI. (Gráfico 2).

Dentro de la información recolectada, 4 casos correspondieron a PTT, con un promedio de sesiones para esta patología de 14.2 (DS 10); 6 casos correspondieron a AHAI para un promedio de 7.3 sesiones (DS 2.3); 1 caso de mieloma múltiple con un promedio de 3 sesiones; 2 casos de lupus eritematoso sistémico con un promedio de 4 sesiones (DS 1.4); 5 casos de esclerosis múltiple, con un promedio de 4.6 sesiones (DS 1.7); 1 caso de glomerulonefritis con un promedio de 6 sesiones; 9 casos de hipertrigliceridemia con un promedio de 2.8 sesiones (DS 0.7); 4 casos de pénfigo vulgar con un promedio de 5.8 sesiones (DS 1.5); 10 casos de Guillain-Barré con un promedio de 6.2 (DS 2.6); 5 casos de miastenia gravis con un promedio de sesiones 3.4 (DS 1.5); 2 casos de hiperviscosidad por enfermedad de Waldeström con un promedio de 2.5 sesiones (DS 0.7) y 1 caso de enfermedad de Wegener con un promedio de 8 sesiones.

Al momento del ingreso a UCI, un 96\% de los pacientes presentó compromiso neurológico, de los cuales lo más frecuente fue la debilidad motora progresiva reportada en un $82 \%$ de los casos; un $86 \%$ ingresó con compromiso ventilatorio que ameritó soporte ventilatorio y un $92 \%$ de 
los casos con compromiso renal ameritando algún tipo de soporte renal. (Gráficos 3, 4, 5). Un $84 \%$ de los casos se egresó de la UCI aún con compromiso neurológico, siendo la debilidad motora progresiva el parámetro que evidencia la mayor mejoría. (Gráfico 3).

Gráfico 2. Porcentaje de pacientes ingresados en la Unidad de Cuidados Intensivos del Hospital Dr. Rafael Ángel Calderón Guardia, a los que se les realizó terapia de aféresis, según número de sesiones, del 01 de Enero del 2010 al 31 de Diciembre del 2012.

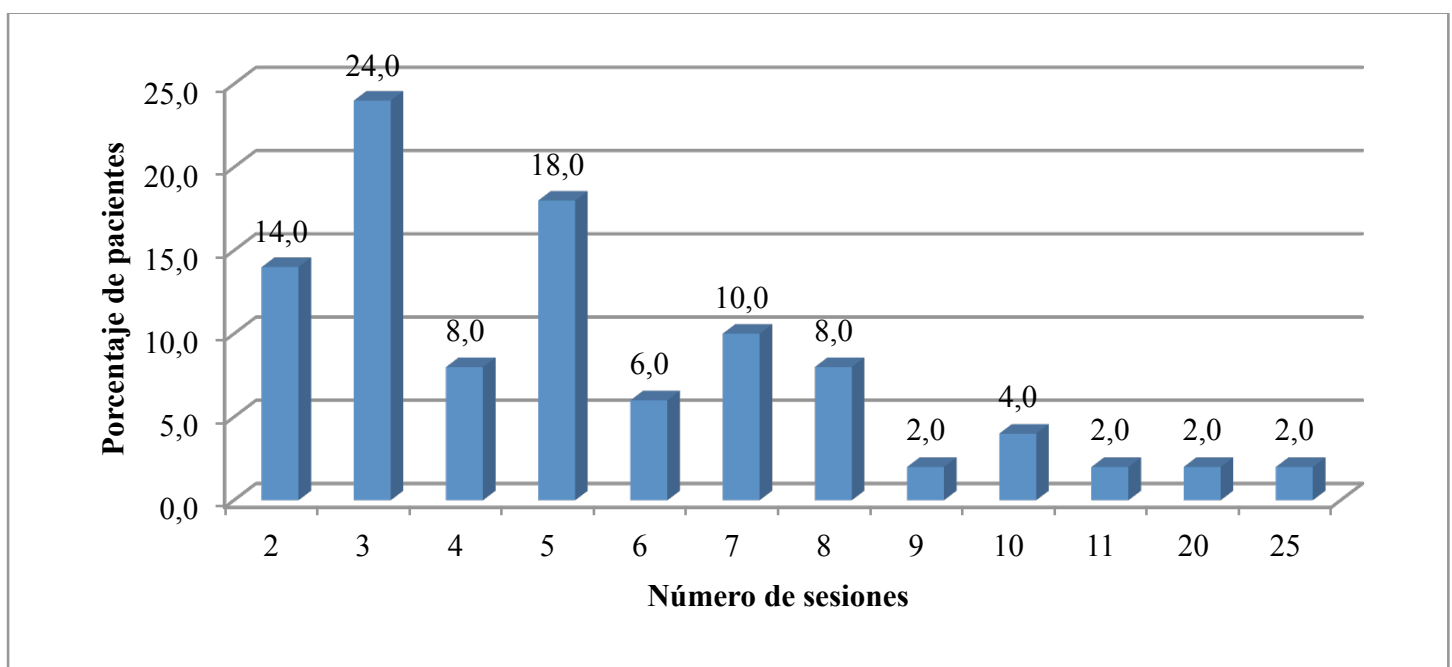

Fuente: Expedientes Clínicos, HCG.

Sí se encontró diferencia estadística significativa $(\mathrm{p}<0.01)$ en las variables cualitativas de ingreso y egreso en lo que respecta al compromiso neurológico.

El 86\% de los pacientes (43 pacientes) requirió soporte ventilatorio al ingreso, al egreso el porcentaje ascendió a 98\% (49 pacientes) incremen- to que no muestra diferencias estadísticamente significativas $(\mathrm{p}=0.06)$. (Gráfico 4$)$. En cuanto a requerir algún tipo de soporte renal, éste varió del $92 \%$ al ingreso (46 pacientes) a $94 \%$ (47 pacientes) al egreso, diferencia no significativa $(\mathrm{p}=10)$.

GRÁFICO 3. Porcentaje de pacientes con Compromiso Neurológico en la Unidad de Cuidados Intensivos del Hospital Dr. Rafael Ángel Calderón Guardia, a los que se les realizó terapia de aféresis, del 01 de Enero del 2010 al 31 de Diciembre del 2012.

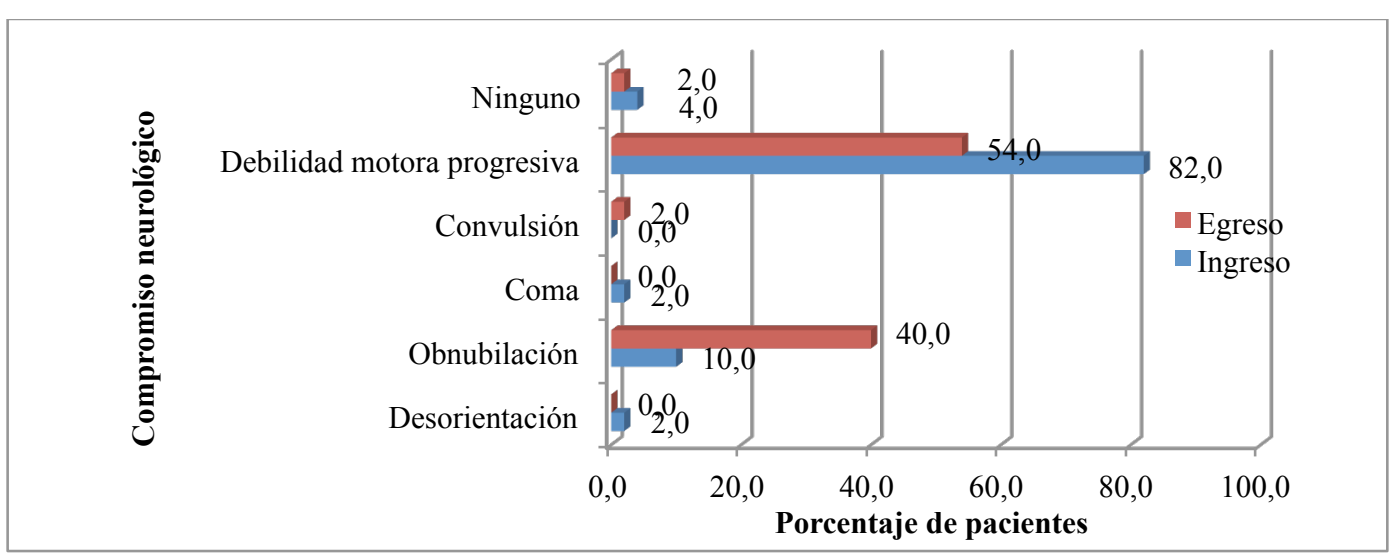

Fuente: Expedientes Clínicos, HCG. 
El 90\% de los pacientes (45 pacientes) ingresó con compromiso hematológico, a su egreso se encontró una mejoría estadísticamente significativa $(\mathrm{p}<0.01)$ ya que el porcentaje se redujo a un $66 \%$ (33 pacientes).

Con respecto a las variables de laboratorio de los pacientes portadores de patologías hematológicas, un 50\% ingresó con niveles de hemoglobina menor a $5 \mathrm{~g} / \mathrm{dl}$. Posterior a la conclusión de las sesiones de aféresis el $26 \%$ de los pacientes persistían con hemoglobinas menores a $5 \mathrm{~g} / \mathrm{dl}$ al egreso, dicha reducción muestra diferencias estadísticamente significativa $(\mathrm{p}=0.004)$. (Gráfico 6).

Un $70 \%$ de los pacientes con enfermedades hematológicas, ingresó con niveles de plaquetas menor de 10000 por $\mu 1$, al egreso el $80 \%$ de los pacientes mantenía los niveles de plaquetas bajos, diferencia no significativa $(\mathrm{p}=0.62)$.

En cuanto a la función renal de estos pacientes, el $18 \%$ (9 pacientes) ingresó con creatininas mayores a $2 \mathrm{mg} / \mathrm{dl}$; no se encontró diferencias significativas $(\mathrm{p}=0.79)$ dado que al egreso el $16 \%$ mantuvieron los niveles iguales que al ingreso. (Gráfico 7).

En $18 \%$ de los pacientes se determinó al ingreso niveles de triglicéridos mayores a $1000 \mathrm{mg} / \mathrm{dl}$, y al egreso un $16 \%$ logró obtener cifras menores a $500 \mathrm{mg} / \mathrm{dl}$; mostrando diferencia estadísticamente significativa $(\mathrm{p}<0.01)$. (Gráfico 8$)$.

En las variables de laboratorio con el análisis estadístico se obtuvo diferencia estadísticamente significativa en parámetros hematológicos, representados por hemoglobina $(\mathrm{p}=0.004)$, reticulocitos $(\mathrm{p}=0.003)$; perfil lipídico, específicamente triglicéridos $(p=0.0003)$. No mostraron cambios significativos plaquetas $(\mathrm{p}=0.62)$, renales mediante documentación de creatinina $(\mathrm{p}=0.79)$ y nitrógeno ureico $(\mathrm{p}=0.99)$.

De los pacientes a los que se le realizó punción lumbar para la obtención de bandas oligoclonales, solamente se pudo documentar la presencia en $22 \%$ de los casos.

La complicación más frecuente asociada al uso de esta terapia fue la hipocalcemia, reportado por un $42 \%$ de los casos, seguido de hipofibrinogenemia con un $8 \%$, luego un $6 \%$ de los casos presento sepsis del catéter y otro $6 \%$ reacción alérgica al plasma. Se documentó en un $2 \%$ de los casos trombosis venosa secundario al uso de un catéter venoso central. (Gráfico 9).

El promedio de días de hospitalización fue de 90.7 días, con una desviación estándar de 130.7 días, y una mediana de 36.5 días de estancia hospitalaria. (Cuadro 1).

GRÁFICO 4. Porcentaje de pacientes con Compromiso Ventilatorio en la Unidad de Cuidados Intensivos del Hospital Dr. Rafael Ángel Calderón Guardia, a los que se les realizó terapia de aféresis, del 01 de Enero del 2010 al 31 de Diciembre del 2012.

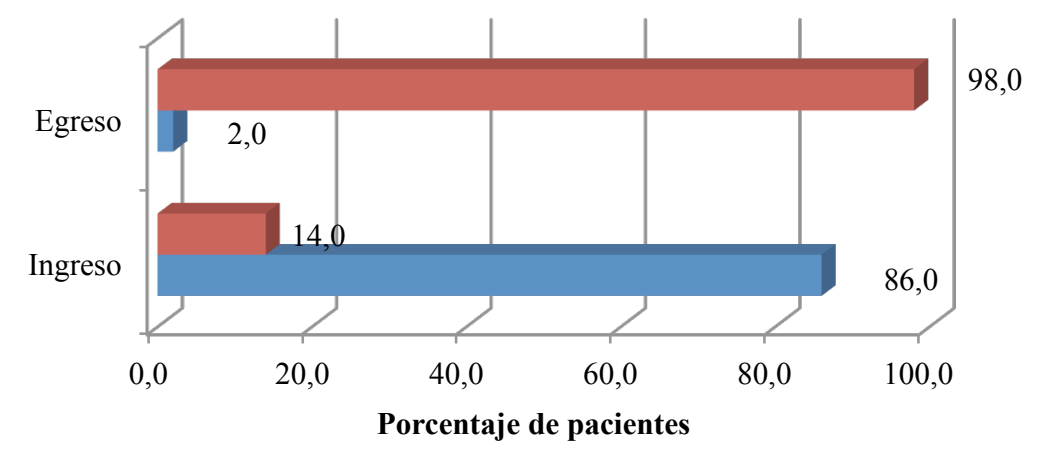

- No soporte Ventilatorio

- Soporte Ventilatorio

Fuente: Expediente Clínico, HCG. 
GRÁFICO 5. Porcentaje de pacientes con Compromiso Renal en la Unidad de Cuidados Intensivos del Hospital Dr. Rafael Ángel Calderón Guardia, a los que se les realizó terapia de aféresis, del 01 de Enero del 2010 al 31 de Diciembre del 2012.

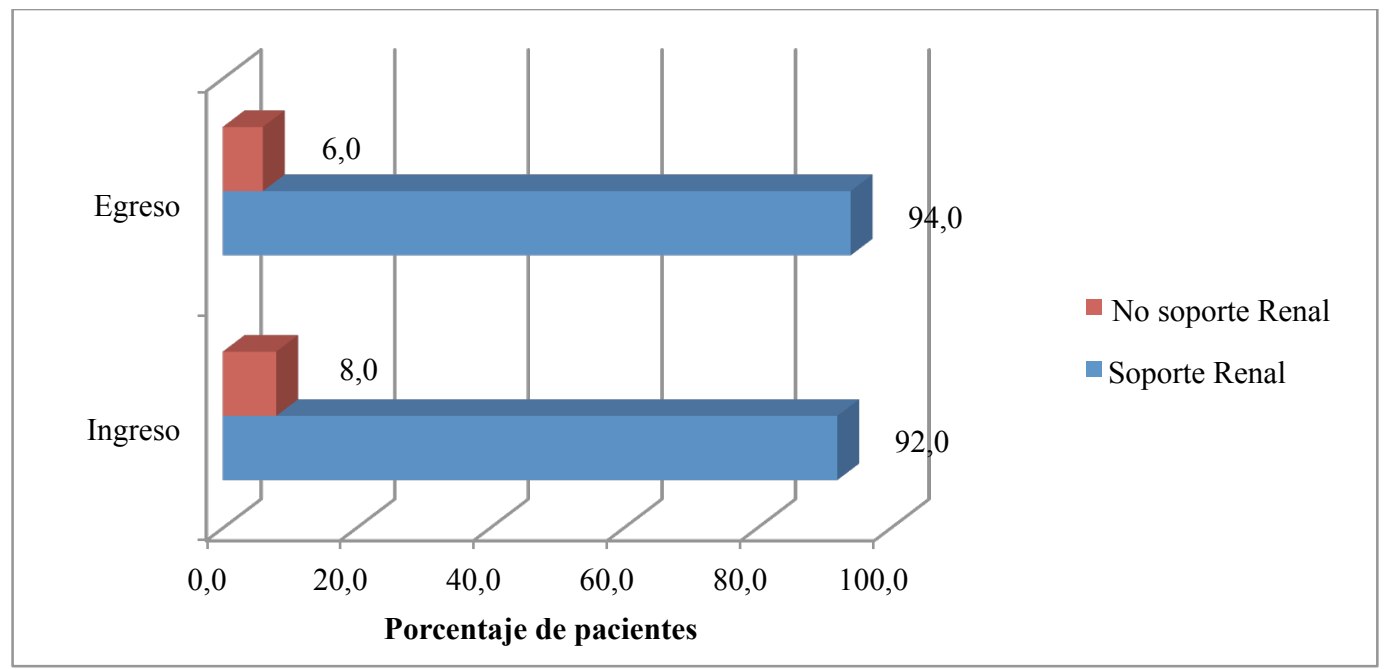

Fuente: Expediente Clínico, HCG

GRÁFICO 6. Porcentaje de pacientes con Compromiso Hematológico en la Unidad de Cuidados Intensivos del Hospital Dr. Rafael Ángel Calderón Guardia, a los que se les realizó terapia de aféresis, del 01 de Enero del 2010 al 31 de Diciembre del 2012.

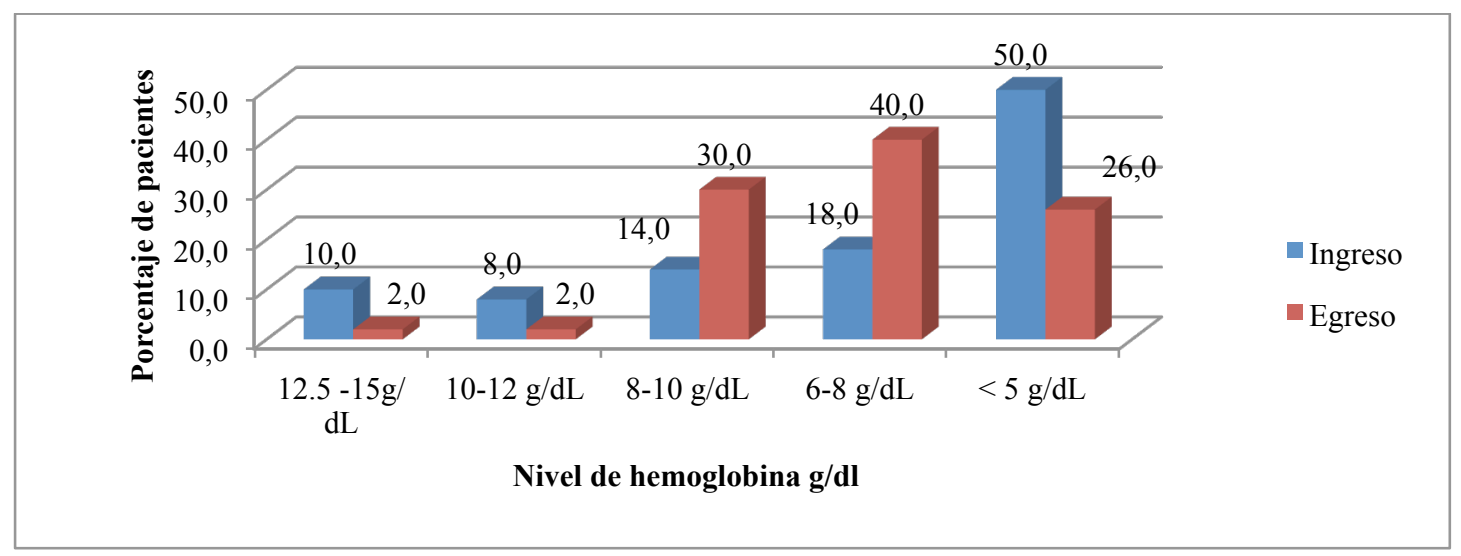

Fuente: Expediente Clínico, HCG. 
GRÁFICO 7. Porcentaje de pacientes con Compromiso Renal en la Unidad de Cuidados Intensivos del Hospital Dr. Rafael Ángel Calderón Guardia, a los que se les realizó terapia de aféresis, del 01 de Enero del 2010 al 31 de Diciembre del 2012.

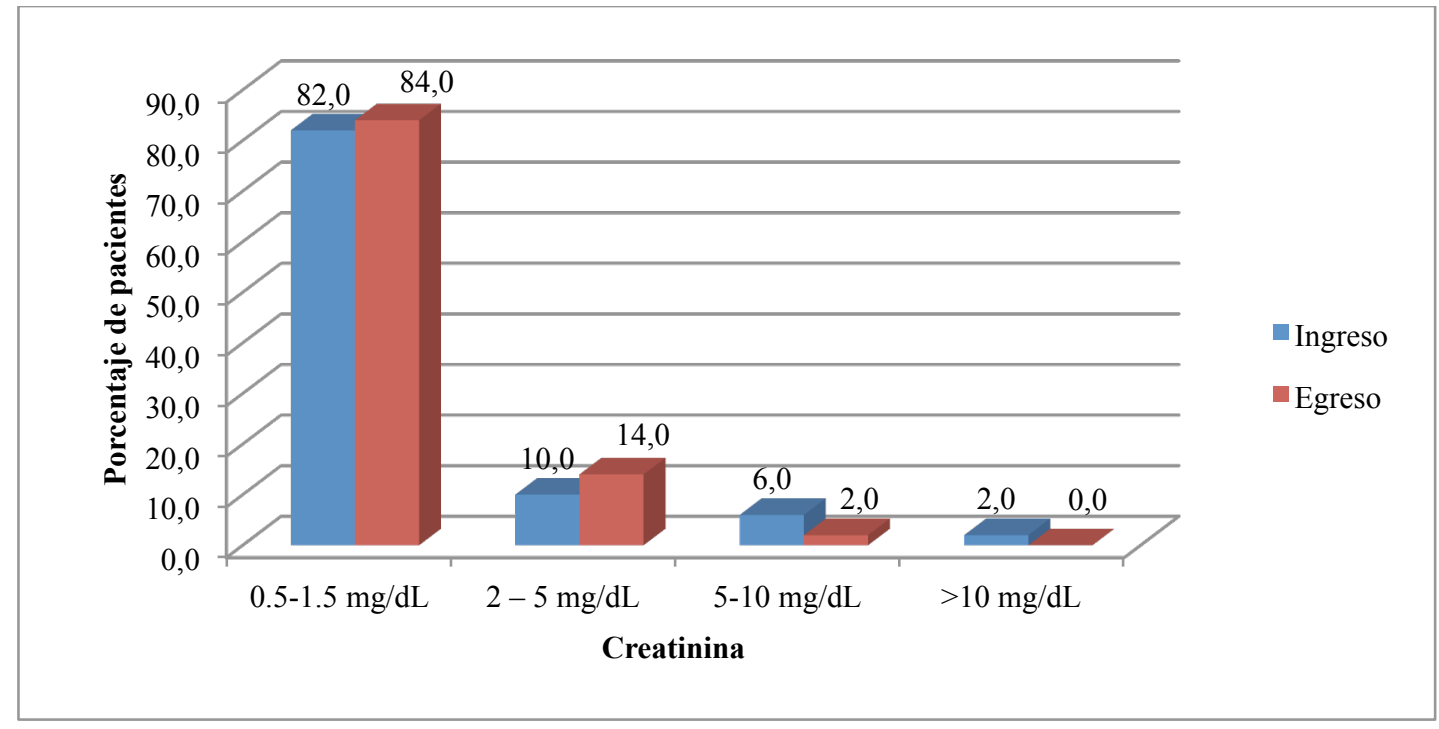

Fuente: Expediente Clínico, HCG.

GRÁFICO 8. Porcentaje de pacientes con Hipertrigliceridemia en la Unidad de Cuidados Intensivos del Hospital Dr. Rafael Ángel Calderón Guardia, a los que se les realizó terapia de aféresis, del 01 de Enero del 2010 al 31 de Diciembre del 2012.

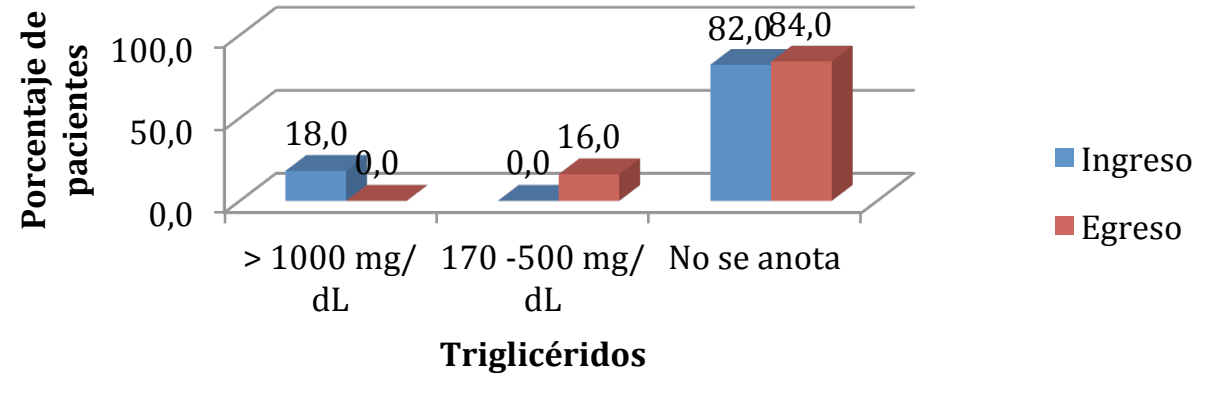

Fuente: Expediente Clínico, HCG. 
GRÁFICO 9. Número de Complicaciones asociadas a la aplicación de la Terapia de Aféresis, en los pacientes ingresados en la Unidad de Cuidados Intensivos, del Hospital Dr. Rafael Ángel Calderón Guardia, del 01 de Enero del 2010 al 31 de Diciembre del 2012.

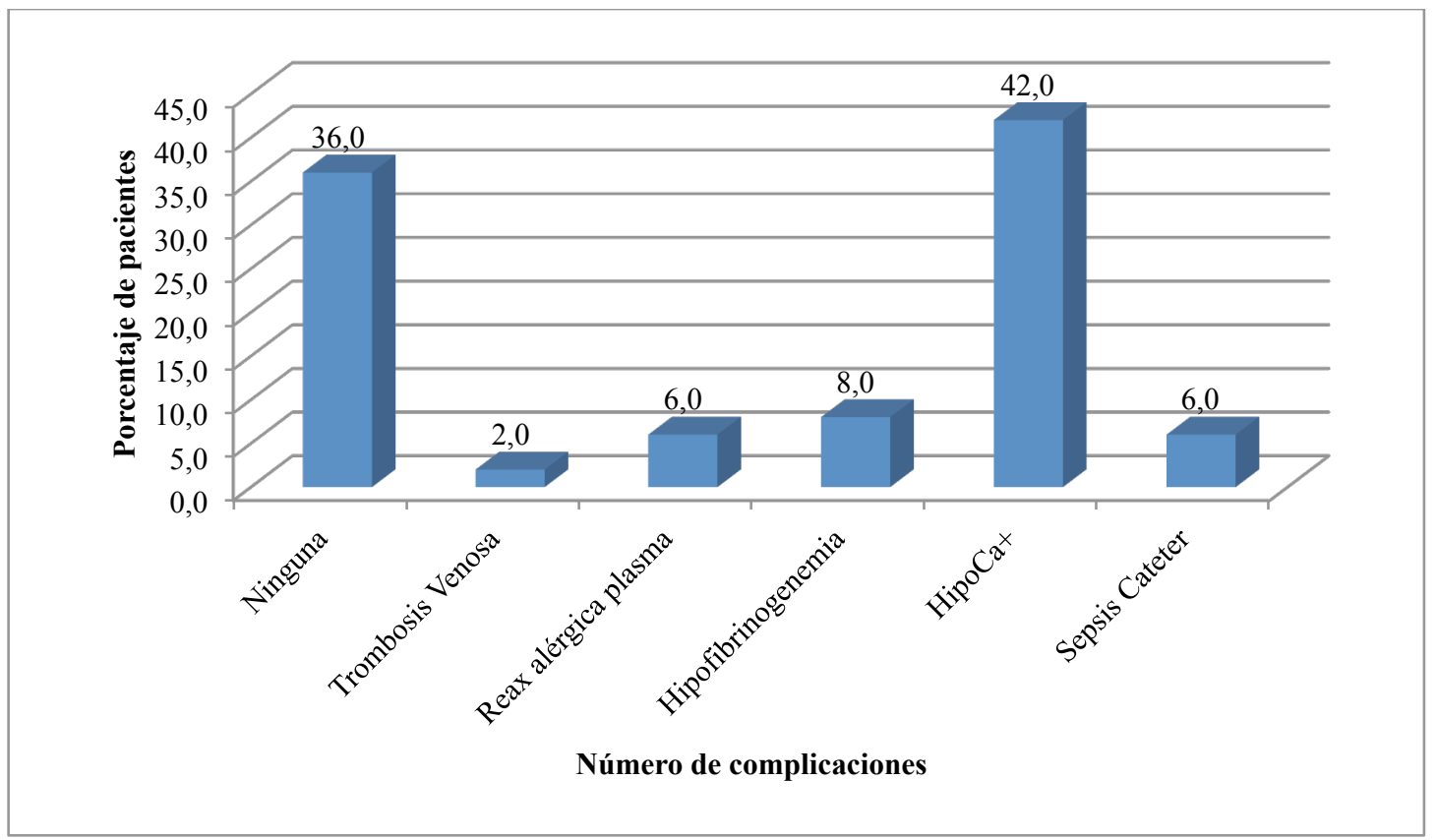

Fuente: Expedientes Clínicos, HCG.

Cuadro 1. Porcentaje de Estancia Hospitalaria en el Hospital Dr. Rafael Ángel Calderón Guardia, del 01 de Enero del 2010 al 31 de Diciembre del 2012.

\begin{tabular}{|ccc|}
$\begin{array}{c}\text { Días de } \\
\text { hospitalización }\end{array}$ & Frecuencia & Porcentaje \\
\hline 1 semana & 6 & 12.0 \\
\hline 2 semanas & 8 & 16.0 \\
\hline 3 semanas & 6 & 12.0 \\
\hline 4 semanas & 3 & 6.0 \\
\hline 2 meses & 7 & 14.0 \\
\hline 3 meses & 3 & 6.0 \\
\hline 4 meses & 4 & 8.0 \\
\hline 5 meses & 4 & 8.0 \\
\hline 6 meses y más & 9 & 18.0 \\
\hline Total & 50 & 100.0 \\
\hline
\end{tabular}

Fuente: Expedientes clínicos HCG

\section{DISCUSIÓN}

En los últimos años, la literatura, basada en el análisis de información de múltiples ensayos clínicos, ha sustentado la eficacia y la seguridad de la aplicación de la terapia de aféresis en diversas patologías de carácter crítico.

En este estudio se documentó la aplicación de la terapia de aféresis en desordenes neurológicos primarios, siendo el más frecuente, con un $20 \%$ de los pacientes, el síndrome de Guillain-Barré y en un cuarto lugar de frecuencia, la esclerosis múltiple y la miastenia gravis, ambas con un $10 \%$.

El segundo lugar de ingresos, se le atribuye a patologías de carácter hematológico, con un 16\% de los casos ingresados correspondiente a anemias hemolíticas autoinmunes, un $8 \%$ en purpura trombocitopénica trombótica y un $8 \%$ por síndrome de hiperviscocidad secundario a mieloma múltiple o enfermedad de Waldeström.

En tercer lugar de frecuencia se incluyen casos de pancreatitis no biliar secundarias a hipertrigliceridemias severas, con una frecuencia del $18 \%$ de los casos.

Se debe recalcar a su vez, haciendo énfasis en la literatura consultada, la utilización de esta terapia en procesos con compromiso inmunológico tales 
como entidades reumatológicas, en donde un $8 \%$ de los casos correspondió a Lupus Eritematoso Sistémico, $2 \%$ a síndrome antifosfolípidos y enfermedad de Wegener respectivamente.

Cabe recalcar, que a pesar de que se aplicó la terapia en casos con compromiso inmunológico severos tales como síndrome antifosfolípidos, enfermedad de Wegener y glomerulonefritis, correspondieron a la minoría de los pacientes ingresados en contraste con lo que la evidencia actual señala.

Desde el punto de vista de eficacia, la mayoría de los pacientes ingresados recibieron pocas sesiones de aféresis, reiterando lo descrito en la bibliografía.

Las patologías con menor número de sesiones por paciente correspondieron a las enfermedades neurológicas, lo que sustenta lo conocido por la literatura mundial, y por ello se deriva su utilización en este ámbito.

Los pacientes portadores de patologías hematológicas, específicamente PTT y AHAI; fueron aquellos que recibieron el mayor número de sesiones, tomando en consideración la utilización concomitante de terapia farmacológica con la aféresis; lo que recalca el carácter refractario de estas patologías a la terapia descrita en la actualidad.

En lo que respecta a las variables clínicas tales como compromiso neurológico, posterior al análisis se documentó la existencia de una diferencia estadísticamente significativa $(\mathrm{p}<0.01)$, lo que podría traducirse como mejoría tras la aplicación de la terapia de aféresis. Siendo el parámetro de debilidad motora progresiva el que reportó mayor beneficio.

Con respecto a las variables cualitativas correspondientes a compromiso respiratorio $(p=0.06)$ y compromiso renal $(p=1.0)$, desde el punto de vista clínico, los pacientes que ingresaron con soporte ventilatorio y renal, independiente de la causa que originara su utilización y tipo de soporte; luego de completar las sesiones terapéuticas de aféresis, continuaron con el mismo. Cabe recalcar que las causas por las que los pacientes requirieron uso de soporte tanto ventilatorio como renal, así como el tipo de soporte utilizado no fueron objetivos en el presente estudio.
En las variables de laboratorio se obtuvo mejoría, representada mediante diferencia estadísticamente significativa, en parámetros hematológicos, representados por hemoglobina y los reticulocitos; así como en perfil lipídico, específicamente los triglicéridos.

En lo que respecta a las patologías con compromiso hematológico, se difirió la inclusión de uso concomitante de terapia transfusional, ya sea por ausencia de información o no requerimiento de la misma.

Las variables de función renal, documentadas por nitrógeno ureico y creatinina, no presentaron mejoría, probablemente sustentado primero por el compromiso multifactorial de ésta y segundo por la complejidad sistémica de la patología que llevó a la afectación del eje renal.

En relación a la seguridad del procedimiento, tal y como se reporta en ensayos clínicos, la aféresis es un procedimiento que puede presentar riesgos o complicaciones: estas se categorizan en leves, en las que no se requiere intervención; moderadas, son aquellas en las que se necesita intervención; severas y fatales, con una frecuencia del $0.8 \%$ y fatales $<0.5 \%{ }^{(7)}$.

Se concluye que la complicación más frecuente reportada en este estudio, correspondió a la hipocalcemia, en un $42 \%$ de los casos; en contraste con lo reportado en la literatura, probablemente atribuible a que este parámetro fue determinado de manera subjetiva y no se logró la determinación cuantitativa del dato.

No se documentó complicaciones severas o fatales.

La repercusión en los días de estancia hospitalaria, se ve determinado por el compromiso clínico de la enfermedad y las complicaciones de la misma, sin lograr correlacionarla con el número de sesiones de aféresis recibidas por paciente

\section{CONCLUSIONES}

La patología más frecuente de ingreso a la Unidad de Cuidados Intensivos para recibir terapia de aféresis, correspondió a la patología neurológica, siendo el Guillain-Barré la de mayor prevalencia. 
Las enfermedades con compromiso hematológico, se posicionan como la segunda causa de ingreso que requiere terapia de aféresis.

La mayoría de los pacientes necesitó pocas sesiones de aféresis, con repercusión en dos esferas: la primera, menor número de complicaciones moderadas y severas; la segunda, disminución en los días de estancia en la Unidad de Cuidados Intensivos no así en la estancia hospitalaria total.

La terapia de aféresis es un procedimiento terapéutico eficaz y seguro, tal y como lo sustenta la literatura a nivel internacional y como se logra demostrar en el presente estudio.

Se encontró mejoría en parámetros clínicos y de laboratorio en la mayoría de los criterios de ingreso de los pacientes al comparar los mismos al egreso de la unidad.

De las complicaciones que más se reportaron al aplicar esta terapéutica en el presente estudio, fueron la hipocalcemia, seguido de la hipofibrinogenemia; descritas ampliamente en la literatura. No se documentó complicaciones mortales

\section{RECOMENDACIONES}

Dado que el presente no es un estudio prospectivo, sería recomendable el diseño de uno con dichas características para aumentar el poder estadístico del mismo.

Incorporar a las variables cualitativas, escalas o parámetros, que permitan un grupo más objetivo y homogéneo para el análisis del mismo.

Incorporar uso concomitante de terapia farmacológica, para poder analizar en términos de eficacia, y comparar ambas terapias.

Desarrollar guías de manejo local que permitan uniformar el abordaje de estos pacientes

\section{REFERENCIAS BIBLIOGRÁFICAS}

1. Balogun R Kaplan A Ward D et al. Clinical Applications of Therapeutic Apheresis. Journal of Clinical Apheresis. 2010;25:250264.

2. Ewald N Hardt P Hans-Ulrich K. Severe hypertriglyceridemia and pancreatitis: presentation and management. Current Opinion in Lipidology. 2009;20:497-504.

3. McLeod B. Evidence based therapeutic apheresis in autoimmune and other hemolytic anemia. Current Opinion in Hematology. 2007;14:647-654.

4. Nguyen T Kiss J Goldman J. The Role of Plasmapheresis in Critical Illness. Critical Care Clinics. 2012;28:453-468.

5. Sanchez A Ward D. Therapeutic Apheresis for Renal Disorders. Seminars in Dialysis. 2012;25:119-131.

6. Szczepiorkowski Z Winters J Bandarenko $\mathrm{N}$ et al. Guidelines on the Use of Therapeutic Apheresis in Clinical Practice-EvidenceBased Approach from the Apheresis Applications Committee of the American Society for Apheresis. Journal of Clinical Apheresis. 2010;25:83-177.

7. Okafor C Ward D Mokrzycki M et al. Introduction and Overview of Therapeutic Apheresis. Journal of Clinical Apheresis. 2010;25:240-249.

8. Winters JL. Apheresis Medicine State of art in 2010: American Society for Apheresis fifth special edition of the Journal of Clinical Apheresis. Journal of Clinical Apheresis. 2011;26(5):239-242.

\section{FUENTES DE APOYO}

No hay fuentes de apoyo que declarar.

\section{CONFLICTO DE INTERÉS}

No hay conflicto de interés que declarar. 